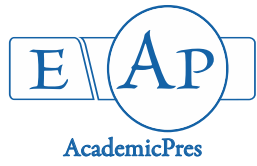

\title{
Green Nanotechnology: Synthesis of Silver Nanoparticles Using Aqueous Leaf Extract of Swertia chirayita
}

\author{
Manoj KUMAR*, Manoranjan P. SINHA \\ Ranchi University, Department of Zoology, Ranchi (Jharkhand), \\ India;dr17mk@gmail.com ("correspondingauthor);dr.mp.sinha@gmail.com
}

\begin{abstract}
In the present study the synthesis of silver nanoparticles mediated by aqueous leaf extracts of Swertia chirayita is reported. Nanoparticles are particles having diameters below $100 \mathrm{~nm}$. The nanoparticles formed were characterized using Uv-Vis spectrophotometer, scanning electron microscope, Ft-IR and light scattering \& Zeta potential analysis. A Surface Plasmon Resonance (SPR) peak was observed at $450 \mathrm{~nm}$ in Uv-Vis analysis. SEM analysis showed that the particles were spherical and cuboidal in shape and had diameter from $85-120 \mathrm{~nm}$. The FT-IR analysis spectra peak were observed at 3,610.74 $\mathrm{cm}^{-1}$, $3,089.96 \mathrm{~cm}^{-1}, 2,125.56 \mathrm{~cm}^{-1}, 166.50 \mathrm{~cm}^{-1}$ and $864.11 \mathrm{~cm}^{-1}$, which corresponds to the presence of capping agents such as primary and secondary amines, hydroxyl compounds, flavonoids, alcoholic and phenolic compounds. The results of light scattering analysis also confirmed the formation of nanoscale particles. The zeta potential analysis showed a peak of $-25 \mathrm{mV}$ which demarcates the stability of the synthesized nanoparticles.
\end{abstract}

Keywords: capping agents, $\mathrm{ft}$-ir, nanoscale, surface plasmon resonance, zeta potential

Abbreviation: FT-IR - Fourier Transform Infrared spectroscopy, SEM - Scanning Electron Microscope, SPR - Surface Plasmon Resonance, Uv-Vis - Uv Visible spectrophotometer

\section{Introduction}

In recent years, appearance of nanometric scale technologies and biological techniques has given rise to a new field called nanotechnology, which focuses on creation and use of materials of nano scale (Goodsell, 2004; Shah et al., 2015). Nanoparticles are of great interest due to their novel physicochemical, magnetic and optoelectronic properties that are governed by their shape and size distribution (Bogunia and Sugiaska, 2002; Kumar et al., 2003). Nanotechnology concerns the size of matters in the range between one $\mathrm{nm}$ to $100 \mathrm{~nm}$ of size. The nano scale imparts ultra-small size, large surface to volume ratio, high reactivity (Dandapat et al., 2014).

Biology of plant-mediated nanoparticles is gaining grounds. The green method of synthesis of nanoparticles has several important applications in the field of biolabelling sensors, drug delivery system. The nanoparticles formed with help of plant extracts exhibit new physico-chemical properties, which are not observed in polar or non-polar extracts of plants (Anil et al., 2013).

Swertia chirayita is an important herb which is commonly available in India, Nepal and China. It is commonly known as Chireta (Hindi). The plant is found at an altitude of $1200-3,000 \mathrm{~m}$ and available throughout the year. It is generally consumed by the older people and people with type II diabetes. It is useful in lowering the blood glucose levels (Dutta et al., 2012).

In this work, we report the synthesis of silver nanoparticles mediated by aqueous leaf extract of Swertia chirayita. This method yields faster and stable silver nanoparticles compared to other methods.

\section{Materials and Methods}

\section{Preparation of plant extract}

$50 \mathrm{~g}$ of sieved leaf powder of Swertia chirayita was subjected to Soxhlet extraction using $350 \mathrm{ml}$ of distilled water. The extract obtained was filtered, concentrated using rotary flash evaporator maintained at $45^{\circ} \mathrm{C}$. The dried extract was stored in airtight containers for further studies (Kumar et al., 2014).

\section{Synthesis of silver nanoparticles}

$1 \mathrm{~mL}$ of Swertia chirayita leaf extract was added 99 to $\mathrm{mL}$ of $1 \mathrm{mM} \mathrm{AgNO} 3(169.8 \mathrm{mg}$ ) solution. The mixture was allowed to stirr for two hrs at $90^{\circ} \mathrm{C}$, during which colour change was observed from light yellow to dark brown. The mixture was allowed to cool down and was centrifuged at 
444

15000 RPM after washing three times with distilled water. A black powder was obtained which was dried overnight in an oven at $80^{\circ} \mathrm{C}$ (Shankar et al., 2004).

\section{Characterization of nanoparticles}

The characterization of silver nanoparticles was done by UV-vis spectrum analysis, SEM analysis, Fourier Transform Spectroscopy (FTIR) analysis and Light scattering \& Zeta potential analysis.

a. UV Visible spectrophotometer (UV-Vis) analysis

The reduction of $\mathrm{Ag}^{+}$ions was monitored by measuring the UV-visible spectrum of the reaction medium after 5 hours by diluting a small aliquot of the sample into Millli-Q water. UV-visible spectral analysis was done by using Parkin Elmer Lambda 25 UV-visible spectrophotometer.

b. Scanning Electron Microscope (SEM) analysis

SEM (Scanning Electron Microscope) analysis was done using JEOL JSM-6390 LV (Japan) machine. Thin films of the sample were prepared on a carbon-coated copper grid by dropping a small amount of the sample on the grid, extra solution was removed using a blotting paper and then the film on the SEM grid was allowed to dry by putting it under mercury lamp for 5 min and was coated with gold using ion sputter.

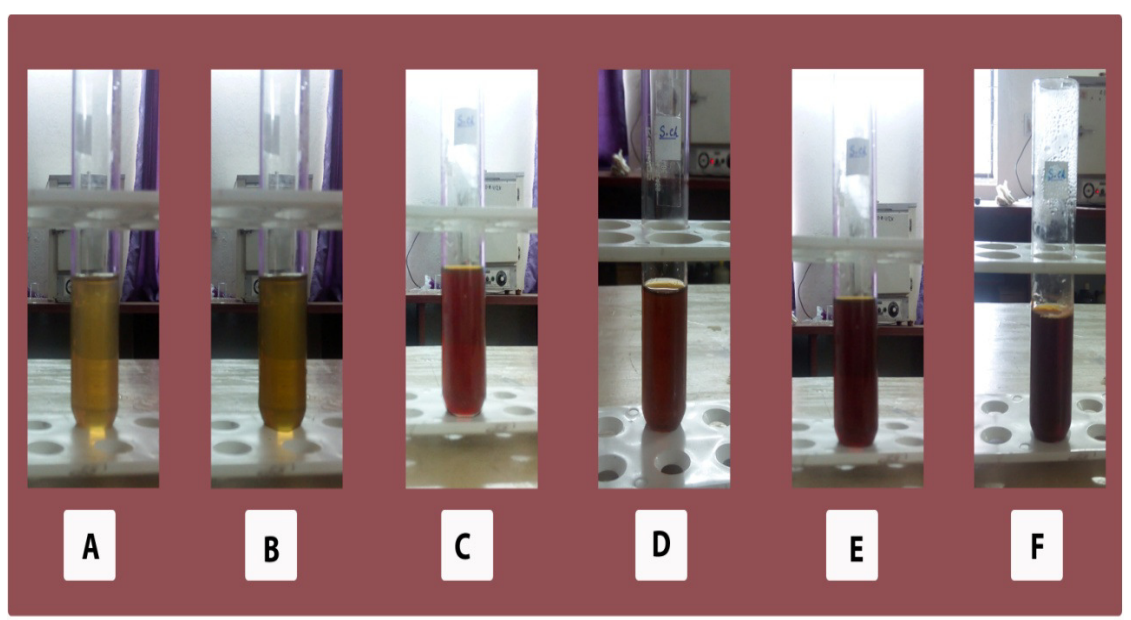

Fig. 1. Colour change from yellow to dark brown due to reduction of silver ions

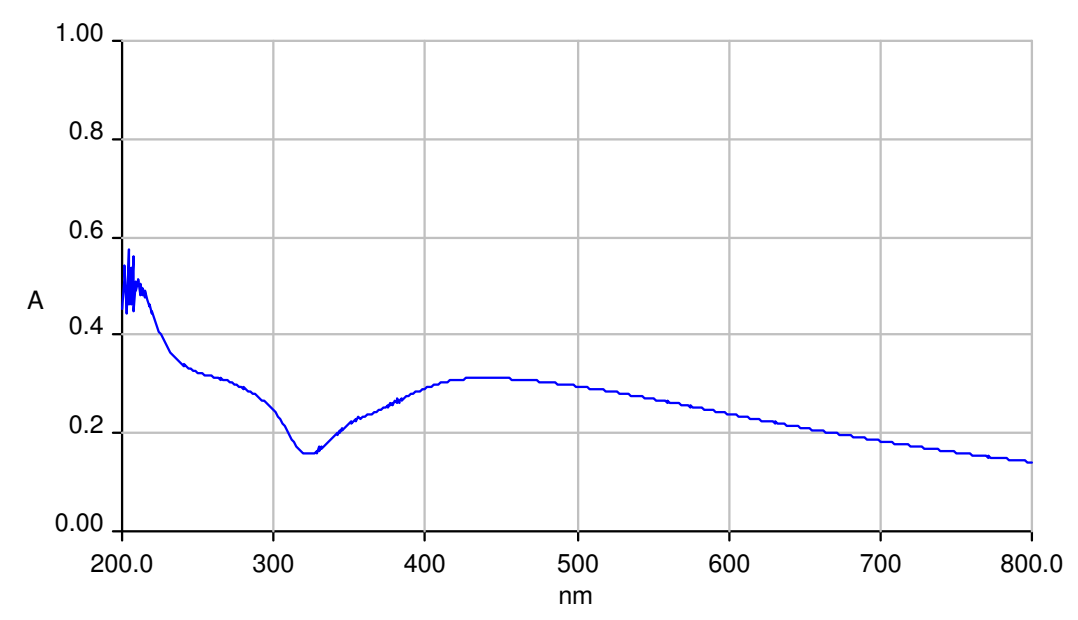

Fig. 2. result of $U V$-vis analysis showing $S P R$ peak at $450 \mathrm{~nm}$ c. Fourier transform infrared spectroscopy (FTIR) analysis

FT-IR analysis was carried out on IP Resting - 21 (Shimadzu) in the diffuse reflectance mode operated at a resolution of $4 \mathrm{~cm}^{-1}$ in the range of $400-400 \mathrm{~cm}^{-1}$ to evaluate the functional groups that might be involved in nanoparticles formation.

d. Light scattering and Zeta Potential analysis

The light scattering and Zeta potential analysis of nanoparticles was carried on Malvern Nano ZS (U.K.).

\section{Results}

\section{$U V$-vis spectrophotometer analysis}

The aqueous solution changed from light yellow colour to dark brown, which is well known confirmation of nanoparticle formation (Shankar et al., 2004; Dhanlakshmi et al., 2012; Kumar et al., 2014). As the aqueous leaf extract of Swertia chirayita was mixed with aqueous solution of $\mathrm{AgNO}_{3}$, a colour change was seen from yellow to brown due to reduction of silver ion (Fig. 1), which indicated the formation of silver nanoparticles. In this study the silver nanoparticles formed showed a (surface Plasmon resonance) SPR peak at $450 \mathrm{~nm}$. The result of UV-vis spectrophotometric analysis is shown in Fig. 2. 
Scanning Electron Microscope (SEM) analysis

The SEM analysis of silver nanoparticles synthesized using aqueous leaf extract of Swertia chirayita revealed the size and structure of the nanoparticles. The silver nanoparticles were spherical and cubical in shape and had diameter of $85 \mathrm{~nm}$ to $120 \mathrm{~nm}$ and average size was $101 \mathrm{~nm}$. The Photographs of SEM is presented as Fig. 3.

Fourier Transform Infrared Spectroscopy (FT-IR) analysis FT-IR analysis was done to determine the role of plant extract as capping agent and the functional groups (Kumar et al., 2014) responsible for synthesis of silver nanoparticles by reducing $\mathrm{Ag}^{+}$ions. The FT-IR absorption spectra of silver nanoparticles are represented as Fig. 4. The spectra showed broad transmission peak at $3610.74 \mathrm{~cm}^{-1}, 3089.96$ $\mathrm{cm}^{-1}, 2125.56 \mathrm{~cm}^{-1}, 1666.50 \mathrm{~cm}^{-1}$ and $864.11 \mathrm{~cm}^{-1}$.

\section{Light scattering and Zeta potential}

The light scattering (also known as static, Rayleigh or Multi-angle light scattering) provides a direct measure of particle size (Satinder and Verma, 2001). The results of light scattering analysis size distribution by number, intensity and volume is represented as Fig. 5(a), Fig. 5(b) and Fig. 5 (c) respectively. The result of Zeta potential analysis is presented as Fig. 6.
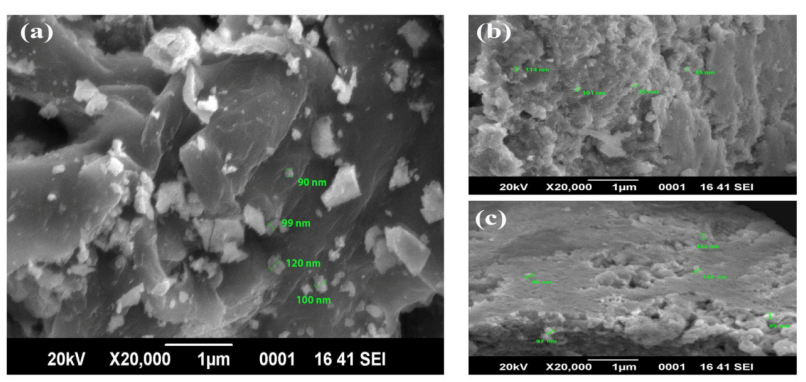

Fig. 3. SEM micrographs showing nanoparticles

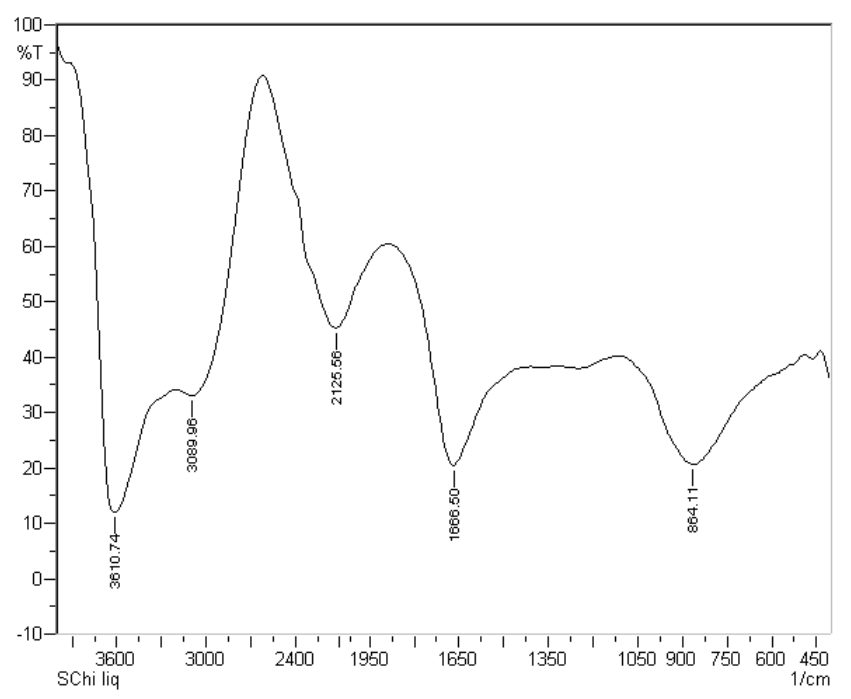

Fig. 4. FT-IR absorption spectra of Silver nanoparticles formed by reduction with aqueous leaf extract of Swertia chirayita

\section{Discussion}

$U V$ Visible spectrophotometer (UV-Vis) analysis

After mixing the Swertia chirayita aqueous leaf extract with the $\mathrm{AgNO}_{3}$ solution, the solution started to change colour from light yellow to brown colour due to reduction of silver ions (Dhanlakshmi et al., 2012; Kumar et al., 2014). The changing of solution from yellowish to dark brown colour has been reported by several authors (Dutta et al., 2012; Anil et al., 2013; Shah et al., 2015) and is a well known confirmation of nanoparticle formation (Satinder $e t$ al., 2001; Bindhu et al., 2013, 2015). The brown colour exhibited is due to the formation of metallic nanoparticles and coherent excitation of all the free electrons within the conduction band, leading to a phase oscillation. The change in colour of solution is presented in Fig. 1. No significant change was observed after $240 \mathrm{~min}$, indicating the end of the reaction (Prabhu and Johnson, 2015). Silver nanoparticles are known to exhibit a plasmon absorption band in the visible region just like gold nanoparticles (Prabhu and Johnson, 2015). From different literature it

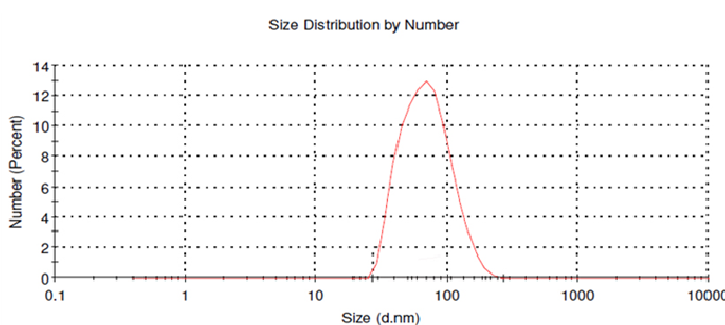

a)

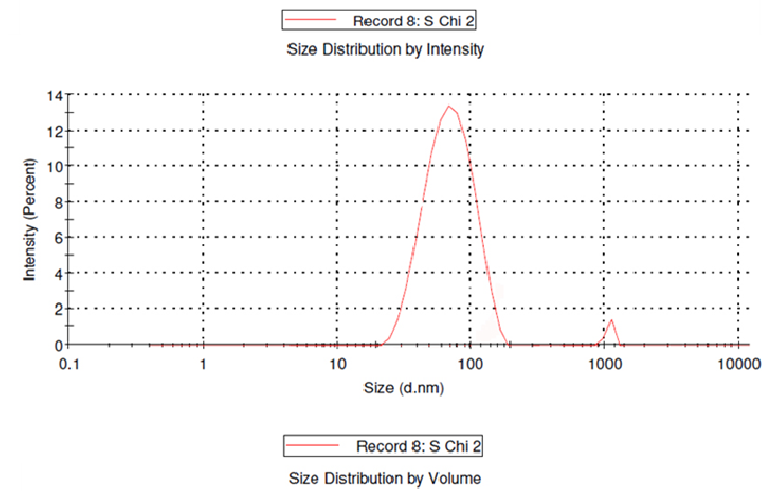

b)

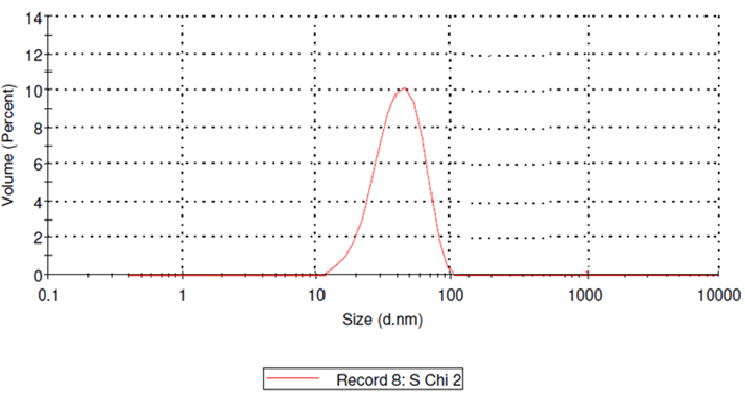

c)

Fig. 5. a) Showing size distribution of nanoparticles by number; b) showing size distribution of nanoparticles by intensity; c) showing size distribution of nanoparticles by volume 


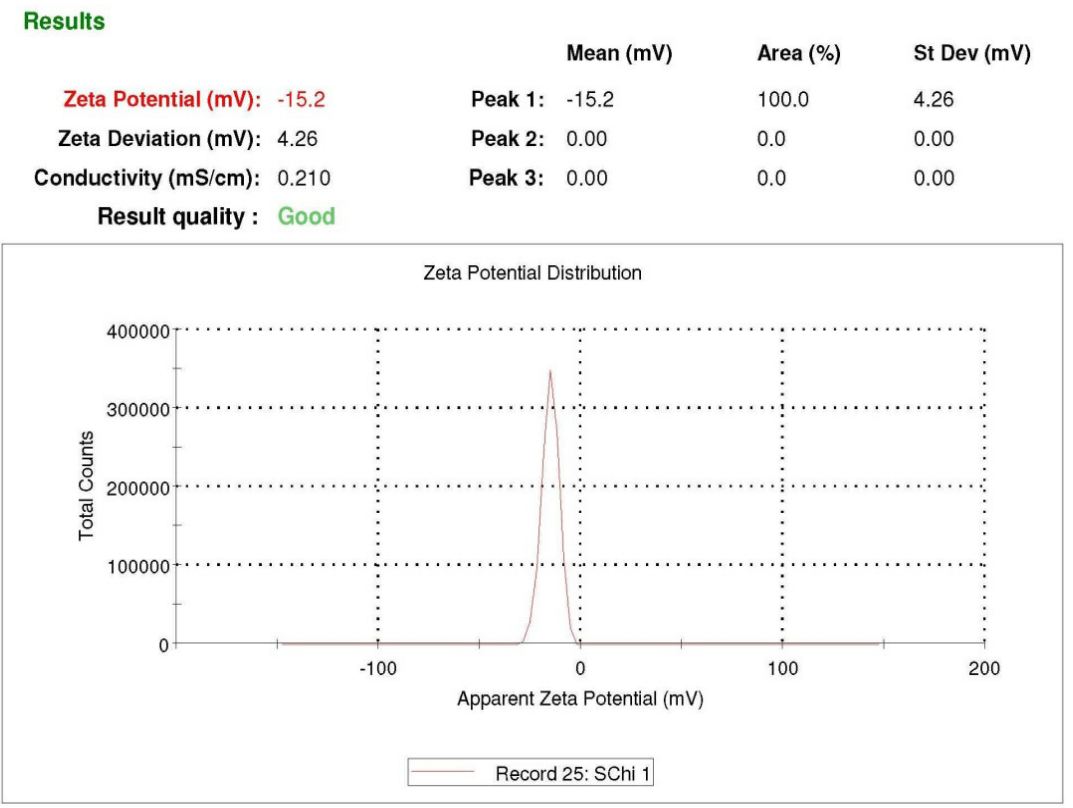

Fig. 6. Results of Zeta Potential analysis

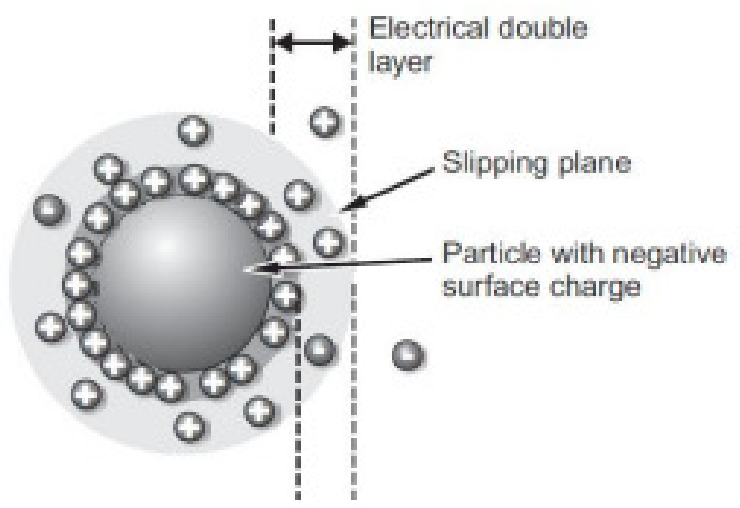

Fig. 7. Electric double layer surrounding nanoparticle [19]

was found that the silver nanoparticles show SPR peak from 415 - 490 nm (Kumar et al., 2003; Anil et al., 2013; Dandapat et al., 2014; Prabhu and Johnson; 2015). A broad peak located at $463 \mathrm{~nm}$ was observed in this study. Prabu and Johnson (2015) reported SPR peak at around $463 \mathrm{~nm}$ for silver nanoparticles synthesized using different plant extracts.

\section{Scanning Electron Microscope (SEM) analysis}

The SEM analysis of silver nanoparticles were done with help of JEOL JSM-6390 LV (Japan) machine. The size of particles in the image can be measured with the help of software provided with the SEM set up on the display monitor. Kumar et al. (2003) reported size of alion mediated synthesized nanoparticles to be in range of 207$293 \mathrm{~nm}$ and the average size was $270 \mathrm{~nm}$. Shah et al. (2015) reported the size of nanoparticles ranging from 20-150 nm in diameter.

\section{Fourier transform infrared spectroscopy (FT-IR) analysis}

The FT-IR absorption spectra of silver nanoparticles showed broad transmission peak at $3610.74 \mathrm{~cm}^{-1}, 3089.96$ $\mathrm{cm}^{-1}, 2125.56 \mathrm{~cm}^{-1}, 1666.50 \mathrm{~cm}^{-1}$ and $864.11 \mathrm{~cm}^{-1}$. FT-IR analysis is done to determine the role of plant extract as capping agent and the functional groups (Pawar and Kamble, 2017). The spectra observed were compared with reference values previously published. The fatty acid stretch was recorded at $1666.50 \mathrm{~cm}^{-1}$ (Pawar and Kamble, 2017). The present study confirms the presence of amines $\mathrm{N}-\mathrm{H}$ (stretch) and C-N (stretch) characteristic peak at $864.11 \mathrm{~cm}$ ${ }^{1}$ (Pawar and Kamble, 2017). The detection of alkaloids is confirmed by the presence of primary and secondary amines (Corlet, 2017). Characteristic peak for hydroxyl compounds -OH (stretch) were obtained $\left(3610.74 \mathrm{~cm}^{-1}\right)$. Detection of hydroxyl groups is an indication of presence of flavonoids, alcoholic and phenolic compounds (Corlet, 2017). The peak at $3089.96 \mathrm{~cm}^{-1}$ corresponds to the $-\mathrm{CH}$ stretching which represents the lipids (Starlin et al., 2012). The peak at 2152.56 corresponds to the $-\mathrm{C} \equiv \mathrm{C}$ - stretching.

\section{Light scattering and Zeta Potential}

The light scattering analysis of nanoparticles is an important tool for characterizing the size of nanoparticles in the solution. It measures the light scattered from a laser that passes through a colloidal solution and by analyzing the modulation of the scattered light intensity as a function of time. The results of light scattering is represented with size distributions of number, intensity and volume.

The result of number distribution is represented as Fig. 5 (a) the figure shows one peak of $93.6 \mathrm{~nm}$ diameter of 
nanoparticles with percentage distribution of $100 \%$. The number distribution shows the number of particles in different size bins (NanoComposix's Guide, 2015). Thus, the result shows that the size of nanoparticles is below 100 $\mathrm{nm}$, i.e. $93.6 \mathrm{~nm}$. The number distribution graph thus shows that, almost $100 \%$ of particles formed had diameter of about $93.6 \mathrm{~nm}$; this confirms the formation of nanoparticles.

The results of intensity distribution is represented as Fig. 5(b), which shows two peaks at $92.5 \mathrm{~nm}$ and $2120 \mathrm{~nm}$ with percentage intensity of $98.2 \%$ and $1.8 \%$ respectively. The intensity distribution describes how much light is scattered by the particles of different size bins (NanoComposix's Guide, 2015). It shows that $98.2 \%$ of light was dispersed by nanoparticles whose average size was $92.5 \mathrm{~nm}$. This shows that about $98.2 \%$ of the particles in the suspension had average size of $92.5 \mathrm{~nm}$. Thus confirms the formation of nanoparticles (particles having diameter below $100 \mathrm{~nm}$ )

The results of volume distribution is represented as Fig. 5(c), two peaks of $78.2 \mathrm{~nm}$ and $2136 \mathrm{~nm}$ is visible with percentage volume of 71.1 and $28.9 \%$ respectively. The volume distribution shows the total volume of particles in different size bins (NanoComposix's Guide, 2015). The volume distribution shows that about $71.1 \%$ of total volume of nanoparticles formed had average diameter of $78.2 \mathrm{~nm}$.

The Zeta Potential analysis is a technique for determining the surface charge of nanoparticles in solution (colloids). Nanoparticles have a surface charge that attracts a thin layer of ions of opposite charge to the nanoparticle surface. This double layer of ions travels with nanoparticle as it diffuses throughout the solution (NanoComposix's Guide, 2012).

The electric potential at the boundary of the double layer is known as Zeta potential of the particle. The Zeta potential of the particles has values ranging from $+100 \mathrm{mV}$ to $-100 \mathrm{mv}$ (NanoComposix's Guide, 2012). The Zeta potential analysis of nanoparticles formed by aqueous leaf extract of Swertia chirayita showed peak of $-15.2 \mathrm{mV}$ with $100 \%$ area distribution (Fig. 6). Nanoparticles with Zeta potential values greater than $+25 \mathrm{mV}$ and less than $-25 \mathrm{mV}$ typically have high degrees of stability (NanoComposix's Guide, 2012). The value of Zeta potential of nanoparticles formed by aqueous leaf extract of Swertia chirayita was found to be in -ve side $(-25 \mathrm{mV})$ which showed the efficiency of the capping material in stabilizing the nanoparticles providing intensive negative charges that keep all the particles away from each other. It implies that the Ag nanoparticle and the solution are stable (NanoComposix's Guide, 2012; Haider et al., 2014).

There are several biological methods of synthesis of nanoparticles (Rajan et al., 2015). The bioreduction potential of plant extracts is comparatively higher than the microbial and fungal methods; many researchers support this hypothesis (Iravani, 2011; Kanan et al., 2011). Researchers have reported the supremacy of green nanoparticles over several applications of polar and non polar plant extracts such as antifungal activity (Dipankar and Murugan, 2012; Singh et al., 2013), antibacterial activity (Gopinath et al., 2012; Satishkumar et al., 2012) and anticancer activities (Satyavani et al., 2011; Jacob et al., 2012).

\section{Conclusions}

On the basis of results obtained by characterization of nanoparticles formed by mediated by aqueous leaf extract of Swertia chirayita by Uv-Vis Spectrophotometer, Scanning Electron Microscopy, Fourier Transform Infrared Spectroscopy and Light Scattering \& Zeta potential analysis; it is clear that nanoparticles (below $100 \mathrm{~nm}$ size) were formed. Thus, we report the formation of $\mathrm{Ag}$ nanoparticles mediated by aqueous leaf extract of Swertia chirayita.

\section{Acknowledgements}

The authors are thankful to the Central Instrumentation Facility (CIF), Birla Institute of Technology, Mesra for helping in FTIR spectroscopy, UVVis spectroscopy and Scanning Electron Microscope (SEM) analysis. The authors also acknowledge the facilities made available by the H.O.D., Department of Zoology, Ranchi University, Ranchi.

\section{References}

Anil KS, Abyaneh MK, Gosavi SW, Sulabha KK, Renu P, Absar (2013). Nitrate reductase mediated synthesis of silver nanoparticles from $\mathrm{AgNO}_{3}$. Biotechnology letter 29:439-45.

Bindhu MR, Umadevi M (2013). Synthesis of monodispersed silver nanoparticles using Hibiscus cannabinus leaf extract and its antimicrobial activity. Spectrochimica Acta Part A: Molecular and Biomolecular Spectroscopy 101:184-190

Bindhu MR, Umadevi M (2015). Antibacterial and catalytic activities of green synthesized silver nanoparticles. Spectrochimica Acta Part A: Molecular and Biomolecular Spectroscopy 135:373-378.

Bogunia-Kubik K, Sugiaska M (2002). From molecular biology to nanotechnology and nanomedicine. BioSystems 65:123-38.

Corlet S (2017). Organic chemistry, Chem 12 A/B Alkaloids functional group worksheet. Available from www.laney.edu $>$ corlet [Last accessed on June $\left.1^{\text {st }}, 2017\right]$

Dandapat S, Kumar M,Sinha MP (2014). Effects of Aegle marmelos (L.) leaf extract and green nanoparticles on lipid profile. The Ecoscan 5 (special issue):157-167.

Dhanlakshmi T, Rajendran S (2012). Synthesis of silver nanoparticles using Tridex procumbens and its antimicrobial activity. Archives of Applied Science and Research 4(3):1289-93.

Dipankar C, Murugan S (2012). The green synthesis, characterization and evaluation of the biological activities of silver nanoparticles synthesized from Iresine herbstii leaf aqueous extracts. Colloids and Surface B: Biointerfaces 98:112-119.

Dutta AK, Gope PS, Makhnoon S, Rahman S, Siddiquee A, Kabir Y (2012). Effect of Solvent extraction on phenolic content, antioxidant and $\alpha$ amylase inhibition activity of Swertia chirayita. International Journal ofDrugDevelopment 4(4):317-325.

Goodsell DS (2004). Bionanomedicine in action. Bionanotechnology: Lessons from nature.John Wiley \& Sons Inc: Hoboken, NJ, USA. 
448

Gopinath V, Mubarak A, Priyadarshni S, Meera P, Thajuddin N, Velusamy $P$ (2012). Biosynthesis of silver nanoparticles from Tribulus terrestris and its antimicrobial activity: a novel biological approach. Colloids and Surface BBiointerfaces 96:69-74.

Haider MJ, Mehdi MS (2014). Study of morphology and Zeta potential analyzer for silver nanoparticles. International Journal of Scientific and Engineering Research 5(7):381-385.

Iravani S (2011). Green synthesis of metal nanoparticles using plants. Green Chemistry 13:2368-2650.

Jacob SIP, Finub JS, Narayanan A (2012). Synthesis of silver nanoparticles using Piper longum leaf extracts and its cytotoxic activity against Hep-2 cell line. Colloids and Surface B: Biointerfaces 91:212-214.

Kanan N, Mukunthan K, Balaji S (2011). A comparative study of morphology reactivity and stability of synthesized silver nanoparticles using Bacillussubtilis and Catharanthus roseus (L.) G. Don. Colloids and Surface B: Biointerfaces 86: 378-383.

Kumar A, Mandal S, Selvakannan PR, Parischa R, Mandale AB, Sastry M (2003). Investigation into interaction between surface-bound alkylamines and gold nanoparticles. Langmuir 19:293-346

Kumar M, Dandapat S, Sinha MP (2014). Plant mediated synthesis of silver nanoparticles using Adhatoda vasica aqueous leaf extract. The Ecoscan 5(special issue):30-36.

NanoComposix's Guide to Dynamic light scattering measurements and analysis, v1.4. February, 2015.

NanoComposix’s Guide to Zeta Potential Analysis of Nanoparticles, v1.1. September, 2012.

Narayan KB, Sakthivel N (2010). Biological synthesis of metal nanoparticles by microbes. Advances in Colloid Interface Science 156:1-13

Pawar S, Kamble V (2017). Phytochemical screening, elemental and functional group analysis of Vitex negundo L. leaves. International Journal of Pharmacy and Pharmaceutical Sciences 9(6):226-230.
Prabu HJ, Johnson I (2015). Plant-mediated biosynthesis and characterization of silver nanoparticles by leaf extracts of Tragia involucrata, Cymbopogon citronella, Solanum verbascifolium and Tylophora ovata. Karbala International Journal of Modern Science 1(4): 237-246.

Rajan R, Chandran K, Harper SL, Yun S, Kalaichelvan P (2015). Plant extract synthesized silver nanoparticles: an ongoing source of novel biocompatible materials. Industrial Crops and Products 70:356-373.

Satinder K, Verma M (2001). Measurement of nanoparticles by lightscattering techniques. Trends in Analytical Chemistry 30(1):417.

Satishkumar G, Gobinath C, Karpagam K, Hemamalini V, Premkumar K, Sivaramakrishnan $S$ (2012). phyto-synthesis of silver nanoscale particles using Moringa citrifolia L. and its inhibitory activity against human pathogens. Colloids and Surface B: Biointerfaces 95:235-240.

Satyavani K, Gurudeeban S, Ramanathan T, Balasubramanian T (2011). Biomedical potential of silver nanoparticles synthesized from calli cells of Citrus colocynthis (L.) Schrad. Journal of Nanobiotechnology 9:43-45.

Shah M, Fawcett D, Sharma S, Tripathy S K, Poinern GEJ (2015). Green synthesis of metallic nanoparticles via biological entities. Materials 8:7278-308.

Shankar SS, Rai A, Anmwar B, Singh A, Ahmad A, Sastry M (2004). Biological synthesis of triangular gold nanoprisms. Natural materials 3(7):482-488.

Singh M, Kumar M, Kalaivani R, Manikandan S, Kumaraguru AK (2013). Metallic silver nanoparticle: a therapeutic agent in combination with antifungal drug against human gungal pathogen. Bioprocess and Biosystems Engineering 36(4):407-415

Starlin T, Arul R, Ragavendran P, Gopalakrishnan VK (2012). Phytochemical screening functional groups and element analysis of Tylophora pauciflora wight and arn. International Research Journal of Pharmacy3(6):180-183. 